\title{
APERTURA AL MUNDO A TRAVÉS DE LA TRADUCCIÓN DE CANCIONES INFANTILES
}

\author{
Opening up to the world through the translation \\ of children's songs
}

\author{
Núria Medina Casanovas* \\ Fecha de recepción: 01/06/2016 • Fecha de aceptación: 21/09/2016
}

Resumen. En este artículo se explica qué son las corales infantiles y qué representan en la enseñanza del ámbito no formal en Cataluña. Seguidamente se hace un repaso del tratamiento de la música en las escuelas de educación primaria de nuestro país. Finalmente se explica la importancia de la traducción de canciones infantiles para el enriquecimiento de la lengua y la cultura, con la incorporación de nuevas costumbres y del vocabulario adquirido a través de la traducción. El artículo explica cuál es el método utilizado para la traducción de canciones infantiles y se pone el ejemplo de dos traducciones del inglés al catalán de canciones del repertorio de las corales infantiles de Cataluña.

Palabras clave: Corales infantiles; Cantaires; Música; Traducción; Rima; Ritmo; Sentido; Naturalidad; Cantabilidad.

Abstract. This article begins by examining the role of that children's choirs played in the realm of non-formal educational institutions and what they represented in Catalonia. We then describe the treatment of music as a subject in the schools of primary education in our country. Finally, we focus on the importance of children's songs being translated and what this implies for the enrichment of the language and culture as well as the incorporation of new traditions and vocabulary. The article also explains the methodology used for the translation of children's songs and gives two translations from English into Catalan that belong to the repertoire of the Catalan children's choirs.

Keywords: Children's choirs; Cantaires; Music; Translation; Rhyme; Rhythm; Sense; Naturality; Cantability.

\footnotetext{
" Departament de Filologia i Didàctica de la Llengua i la Literatura. Facultat d'Educació, Traducció i Ciències Humanes. Universitat de Vic - Universitat Central de Catalunya. C. Sagrada Família, 7. 08500 Vic. Barcelona. España. nuria.medina@uvic.cat
}

Cómo citar este artículo: Medina Casanovas, Núria. «Apertura al mundo a través de la traducción de canciones infantiles», Historia y Memoria de la Educación, 5 (2017): 337-383. 


\section{BREVE RECORRIDO HISTÓRICO}

«Hi ha tanta música al món que és raonable suposar que la música, com la llengua i possiblement la religió, és un tret específic de l'home». ${ }^{1}$

La música forma parte de nuestra vida desde sus inicios. La música es un vehículo imprescindible para el desarrollo intelectual, emocional y espiritual del ser humano. ${ }^{2}$ Podemos decir que una manera de hacer música es cantar. La canción permite acceder a la práctica interpretativa de la música en general de la manera más sencilla, espontánea y natural. ${ }^{3}$ Cantar de manera colectiva, en corales, por ejemplo, favorece la socialización puesto que no se canta de manera individual sino en grupo. Este es el espíritu que impulsa a algunos maestros y pedagogos a la creación de corales infantiles: cantar para compartir experiencias y anhelos.

Las Corals Infantils de Cataluña empezaron en 1962 de la mano de María Dolors Bonal, maestra, pedagoga y fundadora de la coral infantil $L^{\prime} E s q u i t x .{ }^{4}$ Esta coral nació alrededor de la Coral Sant Jordi, en Barcelona. L'Esquellerinc, coral infantil del Cor Madrigal, fue fundada en 1965. El año 1966 se inició el Cor Cabirol, dirigido por Enriqueta Anglada, como filial de la Coral Canigó de Vic. Al mismo tiempo se fueron formando corales infantiles en otras poblaciones como Vilafranca, Lleida, Manresa y Barcelona.

Los directores de las diversas corales organizaron algunas reuniones para hablar de varias cuestiones relativas a ellas. Así es como empezó el Secretariat de Corals Infantiles de Cataluña (SCIC) en 1967. Este movimiento coral impulsó encuentros generales de corales de niños y niñas de 5 a 12 años de toda Cataluña. El primer encuentro de corales infantiles se llevó a cabo en Barcelona.

El movimiento de corales infantiles quiso recuperar todos aquellos aspectos que se consideraban básicos para una educación completa y que estaban prohibidos por la dictadura franquista. Los directores de las

\footnotetext{
${ }^{1}$ John Blacking, Fins a quin punt l'home és músic? (Capellades: Eumo, 1994), 27.

${ }^{2}$ Blacking, Fins a quin punt l'home és músic?, 37.

${ }^{3}$ Joaquim Maideu, Música, societat i educació. (Berga: Amalgama Edicions, 1997).

${ }^{4}$ Carles Capdevila, L'art és un foc d'esperança, per viure, per encomanar i per arreglar el món. Entrevista del periódico ARA a María Dolors Bonal: http://www.ara.cat/videos/entrevistes/Carles-Capdevila-Bonal_3_1525077482.html (consultado el 22 de febrero de 2016).
} 
corales infantiles que iniciaron este movimiento, habían recibido una formación musical en el extranjero, básicamente en Suiza y en Alemania, de donde importaron el método Orff. También aprendieron muchas canciones a base de participar en encuentros de Europa Cantat. Para estos directores la introducción de canciones extranjeras en Cataluña era como abrir una ventana al mundo.

\section{La música en la enseñanza primaria}

El siglo xx ha sido calificado como el siglo de oro de la pedagogía musical por la gran riqueza de aportaciones en esta materia, ${ }^{5}$ con grandes pedagogos como Émile Jaques-Dalcroze, Maurice Martenot, Zoltán Kodály y Carl Orff en la primera mitad del siglo en Europa, y en la segunda mitad del siglo con maestros creativos como Paynter en Inglaterra y Schafer en Canadá, los cuales promueven el desarrollo de la curiosidad sonora de los estudiantes. También hay que destacar a Shinichi Suzuki en el Japón, o Joan Llongueras, Manuel Borgunyó, Ireneu Segarra y Joaquim Maideu en Cataluña. En cambio no ha sido tan rico el tratamiento que la asignatura de música ha recibido por parte de la administración del Estado español, ${ }^{6}$ que, a pesar de gozar de un momento de esplendor en la época de la II República, ha tenido poca consideración en los diversos planes de estudio de la segunda mitad del siglo $\mathrm{xx}$, durante la dictadura de Franco y en la época posfranquista.

Por ejemplo, en los estudios de primaria no se menciona la formación musical de los alumnos hasta la redacción de la Ley General de Educación de 1970. A partir de este momento, las materias que cursaban los alumnos de educación primaria estaban agrupadas en dos grandes bloques: un área de expresión (lengua, matemáticas, plástica, formación musical y educación física) y otro de experiencias con el área de ciencias sociales y naturales y la formación religiosa. Más adelante, con los Programas Renovados (enero de 1981), la música, junto con la plástica,

\footnotetext{
${ }^{5}$ Maravillas Díaz, «La música en la educación primaria y en las escuelas de música: la necesaria coordinación», Revista Electrónica Complutense de Investigación en Educación Musical, 1 (2), (2004): $1-14$.

${ }^{6}$ Núria Medina, English Rap: Una proposta interdisciplinària d'anglès i de música per a Primària. (Tesis doctoral. Universitat de Vic-Universitat Central de Catalunya, 2014).
} 
estaba incluida en el área de Expresión Artística. ${ }^{7}$ Con la aplicación de la Ley de Ordenación General del Sistema Educativo (LOGSE) de 1990, por primera vez una ley abordó una regulación extensa de la música en nuestro país, conectándola con la estructura general del sistema educativo. Con la Ley Orgánica de Educación (LOE) de 2006 se produce una disminución de las horas mínimas destinadas a las enseñanzas artísticas en general, quedando la música con una dedicación de una hora semanal. A pesar de esta situación, la introducción de la llamada sexta hora dio la posibilidad de aumentar las actividades musicales en los centros educativos públicos. ${ }^{8}$ Finalmente, con la aprobación de la Ley Orgánica de Mejora de la Calidad Educativa (LOMCE), Ley Orgánica 8/2913 del 9 de diciembre de 2013, con el aumento de horas dedicadas a asignaturas troncales como las ciencias, las matemáticas y la lengua, condena a la música - considerada por el ministro Wert como una «materia que distrae ${ }^{9}$ - a la práctica desaparición, pasando a ser materia opcional. Con la aplicación de la LOMCE, la impartición de la música y la oferta de enseñanzas artísticas dependen de las comunidades autónomas y de los propios centros educativos, lo cual propicia que haya alumnos que acaben la escolarización obligatoria sin haber estudiado nunca la materia de música. Este es un hecho que no pasa en ningún otro país de la OCDE. ${ }^{10}$

Por este motivo en la formación musical de los niños ha sido imprescindible la enseñanza no formal que, en este sentido, llevan a cabo las corales infantiles en Cataluña a través de la asociación del SCIC. Este movimiento toma como modelo el espíritu de la Escuela Nueva Catalana, con una visión más abierta de la enseñanza.

Después de la Guerra Civil (1936-39) cambiaron las directrices de la pedagogía a las escuelas. Todo el movimiento de renovación pedagógica iniciado en el tiempo de la República quedó anulado con la imposición de un tipo de enseñanza tradicional y conservador. Fue a través de movimientos como el SCIC que algunos de los grandes maestros y pedagogos

\footnotetext{
${ }^{7}$ Díaz, «La música en la educación primaria y en las escuelas de música», 2.

${ }^{8}$ Albert Casals, La cançó amb text improvisat: Disseny i experimentació d'una proposta interdisciplinària per a Primària (Tesis doctoral. Universitat Autònoma de Barcelona, 2009).

${ }^{9}$ En la rueda de prensa concedida el dia 29-06-2012, después del Consejo de Ministros: http://www. eldiario.es/sociedad/profesores-Musica-LOMCE-obligatoria-pierde_0_366063549.html.

${ }^{10}$ Medina, English Rap, 51
} 
de la posguerra pudieron continuar el modelo de enseñanza de la música a través del canto coral tal como propugnaba la Escuela Nueva Catalana. ${ }^{11}$

El SCIC trabaja tanto por la formación de los directores como por la elaboración de material propio, así como en traducciones de repertorio musical. Se procura potenciar a los compositores catalanes, pero también se hacen adaptaciones de canciones extranjeras, incorporando repertorio de fuera que constituye un complemento educativo que, en palabras de María Martorell, «nos parecía imprescindible». ${ }^{12}$

Los directores de corales infantiles en sus inicios eran principalmente maestros que sufrían las mismas prohibiciones que la escuela de los años 60 en Cataluña, como son la privación del uso de la lengua catalana, la coeducación y el conocimiento del propio país. Por este motivo, mediante las corales infantiles querían proporcionar a los niños la posibilidad de conocer y emplear la lengua catalana de una manera natural, compartir las canciones con los niños y las niñas conjuntamente y conocer el país a través de la canción. Algunos de los directores de las Corals infantils fueron discípulos directos de grandes maestros y pedagogos como Artur Martorell o Joan Llongueras. También fueron transmisores de los valores aprendidos en el movimiento Scout, introducido en Cataluña por el padre Antoni Batlle, valores de cultivo cuidadoso y riguroso de la música.

Las corales infantiles beben de las fuentes de las corrientes del mundo coral europeo, como la agrupación Arbeitsgemeinschaft Europäischer Chorverbände (AGEC), fundada el 1955, o Europa Cantat y European Federation of Young Choirs (EFYC), fundadas respectivamente en 1960 y 1963. Precisamente fue Europa Cantat quien encargó la organización del cuarto Festival Europa Cantat Júnior al SCIC, que se celebró en Vic en julio de 2005.

En el mundo de las corales infantiles catalanas se intentó salir al extranjero y contactar con las organizaciones mencionadas, debido a que, en Cataluña, en los años 60, solo había el cancionero del Centro Excursionista de Cataluña, el cual constaba de las traducciones que habían empezado a

\footnotetext{
${ }^{11}$ Núria Medina, Traduccions cantables per a corals infantils catalanes: El repertori anglès-català del SCIC. Trabajo de DEA. Universitat de Vic, 2009.

${ }^{12}$ Expresión extraída de una entrevista personal con la música, pedagoga y en aquellos momentos directora del SCIC.
} 
hacer en la época de los pioneros scouts del padre Batlle. Por este motivo se hizo necesario salir al exterior y comprar cancioneros, sobre todo los que editaba el movimiento Europa Cantat, que fueron traducidos por Oriol Martorell, Montserrat Martorell, Enriqueta Anglada, Josep Vigo y otros directores de corales infantiles. ${ }^{13}$

Para estos autores las canciones ayudan al aprendizaje de la lengua, a la vez que permite a los niños conocer más mundo, puesto que cuando se canta una canción que los cantaires no saben de qué lengua es, se les dice de qué habla. Por eso decimos que ensancha el mundo saber una canción francesa, o una canción tirolesa, o de cualquier otro lugar, porque la letra de la canción te da indicios, o un poco de conocimiento de la cultura de un país. Este hecho se hace principalmente patente si el traductor mantiene las peculiaridades de la letra original de la canción. Pongamos por caso que se traduce una canción tirolesa y la letra habla de la indumentaria de los tiroleses, con calcetas de cuero y un sombrero con una pluma, o bien de la fiesta de la cerveza, esto les da a conocer las particularidades de este país y una apertura al mundo a través de la traducción de canciones de otros países.

Con la educación musical contribuimos a desarrollar la formación integral del niño y su proceso de socialización en varias áreas de desarrollo. ${ }^{14}$ En el ámbito socioafectivo, incentiva el trabajo en grupo, produce satisfacción y placer por la realización de una tarea de grupo bien hecha. Es un medio para fomentar la autoestima, la confianza y la seguridad de los alumnos, sobre todo en el canto coral. ${ }^{15}$ Además, la educación musical desarrolla actitudes de esfuerzo, atención y disciplina.

En el ámbito motor ayuda a definir la lateralidad, mejora la respiración, favorece el desarrollo y conocimiento del esquema corporal. ${ }^{16} \mathrm{La}$

\footnotetext{
${ }^{13}$ Medina, Traduccions cantables per a corals infantils catalanes, 40.

${ }^{14}$ Nikki Rickard, Caroline Bambrick y Anneliese Gill, «Absence of widespread psychosocial and cognitive effects of school-based music instruction in 10-13-year-old students». International Journal of Music Education, 30 (1), (2012): 57-78.

${ }^{15}$ Albert Casals, et al. La concreción de un enfoque didáctico para la educación musical en Primaria. Comunicación presentada al III Congreso de Educación e Investigación Musical. Barcelona 2014.

${ }^{16} \mathrm{~K}$. Marjanen, The Belly-Button-Chord. Connections of pre-and postnatal music education with early mother-child inteeraction. University of Jyväskylä, Finland. Jyväskylä Studies in Humanities, N. ${ }^{\circ}$ 130, Dissertation 2009. Consultado en https:/jyx.jyu.fi/dspace/bitstream/handle/123456789/22602/9789513937690.pdf
} 
música contribuye al desarrollo psicomotor a través de la coordinación de movimientos.

También en el ámbito cognitivo desarrolla la atención y la memoria, la música ayuda a fijar las palabras, amplía la capacidad de comprensión, de análisis y síntesis. Promueve la imaginación y la creatividad, desarrolla habilidades matemáticas y facilita el aprendizaje de idiomas. ${ }^{17}$

Una de las contribuciones esenciales de la práctica de la música en el proceso educativo es la estimulación y modelado de la sensibilidad. La música es un lenguaje artístico. Como lenguaje permite la comunicación (emisión y recepción), y como arte permite la expresión de sentimientos, sensaciones y emociones. ${ }^{18}$

La música, sobre todo la canción que incorpora texto, es una disciplina necesaria en la escuela, porque ayuda a promover el logro de varios aprendizajes, como por ejemplo la lectoescritura. ${ }^{19}$ Varios autores reunidos en el proyecto European Music Portfolio: A Creative Way into Languages (2009-2012) investigaron sobre cómo la música, y más específicamente la canción, ayuda de varias maneras, a la adquisición de la propia lengua. ${ }^{20}$

En este proyecto se explica que, primeramente, la canción desarrolla la comprensión y la expresión oral. El hecho de cantar ayuda en la dicción, la articulación y la claridad en el discurso a la vez que también participa en la mejora de la pronunciación y la fluidez. ${ }^{21}$ En general, la música ayuda a identificar el ritmo y la rima que se pueden encontrar en poemas y canciones. $^{22}$

\footnotetext{
${ }^{17}$ Suzanne Medina, «Using Music to Enhance Second Language Acquisition: From Theory to Practice». En J Lalas \& S. Lee (eds.) Language, Literacy and Academic Devlopment for English Language Learners (Boston: Pearson Custom Publishing, 2002).

${ }^{18}$ Generalitat de Catalunya, Departament d'Educació, Cançons populars i tradicionals a l'escola. Propostes didàctiques i metodològiques (Barcelona: Generalitat de Catalunya, Departament d'Educació, 2012).

${ }^{19}$ Antoni Miralpeix, «La cançó a l'escola: recursos i didàctica» (2012). Accesible en http://www.grups. blanquerna.url.edu/m45/canco (consultado el 10-03-2014).

${ }^{20}$ Karen Ludke y Hanna Weinmann, European Music Portfolio: A Creative Way into Languages- Teacher's Handout. Lifelong Learning Program. COMENIUS-CMP de la Unión Europea (2012).

${ }^{21}$ Karen Ludke, Teaching foreign languages through songs. (Edinburgh: The University of Edinburgh, 2009).

${ }^{22}$ Anna Fomina, «Song Melody Influence on Speech Intonation Memorization», Sixth International Conference on Music Perception and Cognition, eds., C. Woods, G. B. Luck, R. Brochard, S. A. O'Neil, y J. A. Sloboda (Staffordshire, UK: Keele, 2000).
} 
Por otro lado, la canción mejora la comprensión escrita mediante el uso de textos significativos donde se puede encontrar vocabulario y estructuras repetitivas. Al mismo tiempo la canción también mejora la expresión escrita, sobre todo inventando nuevas letras para canciones ya conocidas y así beneficiarse del juego lingüístico. ${ }^{23}$ Además enriquece el vocabulario, puesto que se pueden hacer creaciones de canciones cambiando una palabra por otra e incentivando el uso del diccionario para encontrar las palabras de nueva incorporación, o creando estrofas nuevas siguiendo el ritmo y la melodía establecidos.

Para introducir canciones extranjeras en el repertorio de las corales infantiles catalanas se necesita aplicar la traducción de algunas canciones para hacerlas más cercanas a los niños. En una canción el texto es una parte esencial -aquí entraríamos en la cuestión de si hay que traducir el texto de las canciones o no-. Algunos autores opinan que las corales infantiles de Cataluña tienen que cantar en catalán por varios motivos. Primeramente, porque hace falta que los niños y las niñas entiendan cada una de las palabras que cantan. Después, porque la canción es un vehículo para introducir moderadamente palabras fuera del uso coloquial. Otro motivo importante es que la canción sirve de herramienta integradora para los recién llegados. Finalmente, porque según se dice en una circular del SCIC de 1994, la canción en catalán actúa de contrapeso de la presión de la música de consumo, presente en todas partes, mayormente anglófona.

\section{TRADUCCIÓN DE CANCIONES. MÉTODO DE TRADUCCIÓN}

$\mathrm{Al}$ traducir canciones, Peter Low, traductor de música vocal, establece lo que denomina el principio de Pentatlón. Según Low, ${ }^{24}$ el objetivo de la traducción cantable de un texto es que sea cantado con la música preexistente. Low asegura que la traducción de canciones no se tiene que hacer mediante solo uno o dos criterios, que serían el ritmo y la rima, sino siguiendo cinco criterios. Compara el traductor de canciones con el atleta olímpico de pentatlón. Mediante esta metáfora, Low afirma que el traductor de una canción tiene cinco pruebas en las cuales hay que com-

\footnotetext{
${ }^{23}$ David Crystal, How Language Works (London: Penguin Books, 2007).

${ }^{24}$ Peter Low, «The Pentathlon Approach to Translating Songs», en Song and Significance: Virtues and Vices of Vocal Translation, ed. Dinda Gorlée (Amsterdam-New york: Rodopi, 2005).
} 
petir, cinco criterios de traducción que hay que conseguir, con el fin de obtener el mejor resultado final. Los cinco criterios son: el ritmo, la rima, el sentido, la naturalidad y la cantabilidad (singability).

Las canciones infantiles, al tener mucho más ritmo y rima, la letra es más fácil de asumir. Hace falta sobre todo imbuirse del ritmo musical, ver dónde caen los acentos es lo más importante. Lo primordial es que coincida el ritmo musical con el ritmo prosódico de la palabra, y sobre todo evitar que los acentos caigan de modo inadecuado. Cuando se traducen canciones hay que buscar que haya rima en el texto de llegada si es posible, pero no a costa de otros aspectos como, por ejemplo, el significado o la cantabilidad que generalmente son prioritarios.

Sentido: que exista una relación semántica entre la lengua de salida y la lengua de llegada. Buscar las palabras que sean lo más parecidas. Interiorizar y memorizar la canción; es importante lo que dice y cómo lo dice.

Naturalidad, sobre todo en el registro y el orden de las palabras. En el caso de canciones infantiles las traducciones utilizan palabras sencillas propias del vocabulario infantil, a pesar de que a veces algunos traductores, como Enriqueta Anglada o Josep Vigo, introducen adrede palabras poéticas o cultas que consideran que ayudan a enriquecer el vocabulario de los niños. ${ }^{25}$ Los dos traductores de canciones infantiles defienden que hay que enriquecer el vocabulario, con el único matiz de que hay que huir de la utilización de giros poco naturales en la lengua de llegada y descartar arcaísmos o palabras de que los niños no comprendan su significado. Los traductores defienden la utilización de un registro culto o poético a la hora de escoger el vocabulario. De este modo colaboramos a mantener la riqueza de la lengua y a no empobrecerla, procurando utilizar expresiones y frases hechas propias de la riqueza de nuestra lengua.

Cantabilidad, es decir, que el texto pueda ser cantado. Por eso a la hora de traducir hay que trabajar el texto escrito y la música simultáneamente. Por ejemplo, hay que tener en cuenta dónde caen las vocales del texto y resaltar algunas palabras en el mismo lugar donde resaltaban en el texto de salida. También es imprescindible que una música alegre vaya acompañada de una letra alegre, puesto que las palabras reflejan la

\footnotetext{
${ }^{25}$ Medina, Traduccions cantables per a corals infantils catalanes, 60 .
} 
música y lo que esta expresa. Hace falta sobre todo producir una letra que sea comprensible y que suene natural cuando se cante.

\section{ANÁLISIS DE CANCIONES}

A continuación presento la traducción de dos canciones. ${ }^{26}$ Una canción traducida por Josep Vigo en 1994: Cuckoo! en inglés, Cucut! en catalán (Anexo 1), y otra canción traducida por Enriqueta Anglada: Tommy and the Apples en inglés, El pomer en catalán, traducida en 1991 (Anexo 2). La de Vigo es una canción a dos voces de Benjamin Britten, y la de Anglada, una canción de cuatro estrofas tradicional de Gales. Seguiré los criterios de ritmo, rima, sentido, naturalidad y cantabilidad del llamado principio de Pentatlón descrito por Peter Low ${ }^{27}$ para analizarlas.

Hay que tener en cuenta que la explicación que se hace de las canciones se dirige a los niños y niñas cantores, llamados cantaires y directores de corales infantiles, para poder hacer una introducción pedagógica y musical de las canciones que se presentan a los chicos y chicas, procurando de este modo una apertura al mundo de los niños a través de la traducción de las canciones infantiles.

\section{COCKOO!/ CUCUT!}

\section{Ritmo}

La letra del texto inglés respeta los acentos musicales: In April l'abril; In May el maig; y se sirve del comienzo anacrúsico para mantener la onomatopeya del sonido del cuco, dando así el acento a la segunda sílaba: cuckoo cucut, tal como corresponde a dicho sonido. La segunda voz repite el ostinato Cuckoo, cucut, manteniendo la onomatopeya y el acento musical coincidiendo con el acento prosódico en la segunda sílaba.

La canción mantiene mayoritariamente en todos los versos un número idéntico de sílabas.

Ejemplo: In August away I must

Sabeu doncs què faig? Me'n vaig.

\footnotetext{
${ }^{26}$ Medina, Traduccions cantables per a corals infantils catalanes, 81-105

${ }^{27}$ Low, «The Pentathlon Approach to Translating Songs».
} 


\section{Rima}

En el texto de la canción cada uno de los versos tiene una palabra al comienzo y al final de cada verso que riman:

Cuckoo, Cuckoo, What do you do? Cucut, cucut! Plou i és eixut.

In April I open my bill;

L'abril, ves!, fa poc que n'he après.

In May I sing night and day;

El maig ja canto a bell raig.

In June I change my tune;

El juny tothom qui em sent

In July far-far I fly;

In August away I must.»

diu que entono malament.

Cuckoo, cuckoo, cuckoo!

Sabeu, doncs, què faig? Me'n vaig.

Cucut, cucut, cucut

\section{Sentido}

Antes de cantar esta canción, se pueden trabajar con la coral infantil los temas siguientes: el tiempo de la llegada del cuco en Cataluña, que es a principios de abril, ${ }^{28}$ concretamente el 3 de abril es la fiesta que popularmente se asigna a la llegada de este pájaro, considerado como el que anuncia la primavera. El diccionario también nos cita algunos refranes, y más adelante nos cuenta que se relaciona el cuco con la llegada de lluvias: Al temps del cucut, al matí moll i al vespre és eixut, dicho popular de Pont de Suert Tremp, Pel temps del cucut, del matí al vespre és eixut en Olot. Por eso el traductor utiliza la expresión Plou $i$ és eixut, refiriéndose al dicho popular.

En L'abril, ves!, ves se usa como exclamación de extrañeza. Otro motivo por el cual se usa esta expresión es para implicar al público que lo cantará, y posiblemente para añadir una sílaba.

En Ja canto a bell raig, la canción hace el símil de la fuente con el canto: cuando una fuente tiene un bell raig, quiere decir que es una fuente con un chorro de agua abundante.

\footnotetext{
${ }^{28}$ Antoni Maria Alcover y Francesc de Borja Moll, Diccionari Català-Valencià-Balear (Palma de Mallorca: Moll, 1985).
} 
El cuco hace un monólogo y nos explica lo que hace en los meses de abril, mayo y junio, o sea, por la primavera. El cuco habla solo y él mismo se hace preguntas y respuestas.

Cantar y entonar pueden ser sinónimos, pero entonar tiene connotaciones negativas, en este caso diu que entono malament. Britten cuida mucho la relación entre letra y música. Por eso cuando hay un cambio en la melodía la letra dice I change my tune, aunque esto quizás no quiere decir que entone mal, sino simplemente que lo hace de modo diferente. Si miramos la partitura, o bien seguimos la melodía de la canción, veremos que cuando dice I change my tune la melodía es diferente. Cuando la letra de la canción explica que el cuco se va hay un nuevo cambio y la melodía sugiere el vuelo de un pájaro.

La traducción nos aclara el sentido de I open my bill traduciéndolo por fa poc que n'he après. En la versión inglesa dice I change my tune; en la traducción este cambio de canto queda más específico y nos explica que entono malament.

La canción en inglés nos habla de cinco meses del año, cuando en la traducción al catalán habla en concreto de los tres meses de la primavera, posiblemente por concisión. La canción catalana introduce datos meteorológicos que nos dan más información, dice que plou $i$ és eixut.

En el original inglés, la canción introduce dos personajes. En cambio, el cuco de la versión catalana habla a solas. Aun así, antes de acabar el monólogo pregunta a un posible oyente: Sabeu, doncs, què faig?, y sin esperar respuesta ninguna, él solo da la solución: Me'n vaig.

\section{Naturalidad}

Esta canción nos explica el ciclo vital del cuco. Es una canción pastoril. La versión en inglés nos recita en forma de diálogo una conversación entre el pájaro y otro personaje, probablemente un pastor. En esta conversación el cuco nos cuenta qué hace durante los meses de primavera y de verano. Cuando el verano ya está a punto de acabar el ave tiene que emigrar hacia tierras más cálidas Por eso dice away I must.

En catalán, en cambio, esta forma de diálogo que habíamos visto en inglés toma una perspectiva diferente. Ahora es el cuco el que por volun- 
tad propia nos explica, a manera de declamación, lo que hace. Esta vez, no obstante, el traductor solo nos informa de tres meses: abril, mayo y junio. Del texto catalán se deduce que el cuco emigra hacia tierras más cálidas a partir de junio, aunque sabemos que no es hasta el otoño cuando los pájaros marchan hacia otras tierras buscando temperaturas más suaves. En este caso el texto se subordina a prioridades rítmicas y de rima. El texto sigue la sucesión abril, mayo, junio y, por cuestiones de ritmo, el autor del texto catalán ya no puede mencionar más meses.

Así, pues, la estructura pregunta-respuesta cambia en la traducción. El recurso que le queda al autor de la traducción es hacer que el propio cuco se pregunte a sí mismo y responda: Sabeu, doncs, què faig? Me'n vaig. De este modo, por mecanismo de compensación, recuperamos la pregunta.

El traductor da importancia a la naturalidad de registro y se sirve de refranes populares, como, por ejemplo, utilizar una parte del refrán popular el temps del cucut: tan aviat plou com és eixut en el primer verso.

Vale la pena anotar el hecho de que la expresión inglesa night and day en catalán no sería noche y día, sino día y noche. Aquí, pues, el uso de la lengua nos hace comprender la diversidad de los idiomas.

Existe una relación semántica entre el texto de salida y el texto de llegada.

Ejemplo: In May I sing night and day

\section{El maig ja canto a bell raig}

Si el texto inglés nos hace una descripción de todo el ciclo vital del cuco, el texto catalán solo nos hace una referencia al canto del pájaro.

Comparamos los tres meses que ambas versiones recogen.

Abril:

Texto inglés: I open my bill (abro el pico).

Texto catalán: fa poc que n'he après (hace poco tiempo que he aprendido). 
Aquí el texto catalán queda muy ambiguo; o sea, no sabemos qué es lo que ha aprendido. ¿Ha aprendido a comer?, ¿a abrir el pico?, ¿a volar? , ¿a cantar? La respuesta a estas preguntas la sobreentenderemos cuando el traductor nos hable del mes de mayo.

Mayo:

Texto inglés: I sing night and day (canto de día y de noche).

Texto catalán: ja canto a bell raig (canto muy fuerte).

Ambos textos coinciden con el hecho de cantar, pero en inglés nos explica cuándo canta, mientras que en catalán nos explica cómo canta.

Junio:

Texto inglés: I change my tune (yo cambio el tono).

Texto catalán: tothom qui em sent diu que entono malament (todos los que me escuchan dicen que canto mal).

La versión original inglesa dice que cambia de tono, no para entonar mal, tal como especifica la traducción, sino simplemente como cambio. El traductor no recupera en ningún momento de la traducción los meses de julio y agosto, que ni siquiera menciona.

Finalmente, parece como si el cuco, ofendido por los comentarios negativos de la gente en cuanto a sus cantos, se va. Este final es una introducción libre que ha aportado el traductor, consiguiendo así una rima interna del texto que no existía en la versión original.

\section{Cantabilidad}

Resumiendo, mientras que Jane Taylor, a través de una interrogación al cuco, nos cuenta todo lo que este pájaro hace durante los meses de primavera y verano, Josep Vigo nos muestra un canto de lamento por parte del mismo cuco. El texto de Jane Taylor es más descriptivo que el de Josep Vigo, que nos introduce los sentimientos del ave.

Josep Vigo mantiene los acentos musicales en concordancia con los acentos prosódicos, a la vez que procura que el texto mantenga la rima interna que el texto de Jane Taylor propone. La naturalidad con que se 
desarrolla el texto da un argumento a la historia del cuco, y hace que el cantaire encuentre en la canción el aspecto de la cantabilidad muy conseguido en la traducción al catalán. El autor del texto en catalán resalta algunas palabras en el mismo lugar donde resaltaban en el texto original. Ejemplos: cucut, abril, maig, juny.

\section{TOMMY AND THE APPLES/ EL POMER}

\section{Ritmo}

Al ser esta una canción con estrofas, en algunas de ellas, tanto en inglés como en catalán, los autores se sirven del comienzo anacrúsico para poder incluir toda la letra respetando los acentos prosódicos y musicales a la vez. El texto catalán empieza la primera estrofa con comienzo anacrúsico: así el acento principal recae en la segunda palabra Jaume, y este comienzo anacrúsico sirve para el artículo En. Esta misma estrategia se sigue en el texto catalán para el último verso de la primera estrofa.

El texto original inglés utiliza el comienzo anacrúsico para la entrada de la cuarta estrofa: Now boys, y también para los últimos versos de las estrofas segunda y cuarta. Segunda estrofa: And Tom came tumbling after; cuarta estrofa: And that would be a pity. También utiliza el ritmo anacrúsico para el segundo verso de todas las estrofas, excepto para la primera. Segunda estrofa: The tree it shook with laughter; tercera estrofa: The apples in a basket; cuarta estrofa: For ending of my ditty.

La estructura de la música es una combinación de una negra y una corchea a cada tiempo. De este modo se obtiene un ritmo con sensación de canción infantil.

Ejemplos: And as Tom was falling down

\section{Mentre en Jaume va caient}

Took them to the market town

i les portarà al mercat

\section{Rima}

En el texto inglés, los versos de cada estrofa riman el segundo con el quinto; en el catalán, se repiten las palabras, excepto en la tercera estrofa, en la que las palabras del segundo verso y el quinto no riman: pomes, vila. 


\begin{tabular}{llllll} 
& \multicolumn{2}{c}{ En inglés } & & \multicolumn{2}{c}{ En catalán } \\
\cline { 2 - 3 } \cline { 5 - 6 } 1. a estrofa: & apples & apples & pomes & pomes \\
2. ${ }^{\text {a }}$ estrofa: & laughter & after & $\varnothing$ & $\varnothing$ \\
3. ${ }^{\text {a }}$ estrofa: & basket & market & $\varnothing$ & $\varnothing$ \\
4. ${ }^{\text {a }}$ estrofa: & ditty & pity & viure & riure
\end{tabular}

El texto en catalán consigue los monosílabos del cuarto verso en cada estrofa, excepto en la tercera, donde, para conseguir un efecto similar, repite la última sílaba de la palabra anterior, en este caso mercat, cat, cat. La autora del texto en catalán consigue con esta repetición un aire infantil.

\section{Sentido y Naturalidad}

Esta es una canción con una moraleja final; una lección para los niños que dice lo que no se debe hacer, a pesar de que se utiliza un tono humorístico. A comienzos de la cuarta estrofa en inglés dice: «Escucha bien mi consejo»; y en catalán: "Aprended bien la lección»; en inglés: «No trepes muy alto que te caerás»; y en catalán: «Quien está embobado se cae».

La canción formada por cuatro estrofas es la narración de un hecho que le sucede a un niño, Tommy, en el texto de salida, y Jaume en el texto de llegada. Ya solo al empezar la primera estrofa la versión catalana nos muestra un personaje poco vivaracho: dice que és babau palabra que rima con cau. Esta cuestión no forma parte del original, puesto que en el texto inglés si el protagonista cae no es por culpa suya sino del árbol y del musgo que ha crecido con la consecuente viscosidad que hace que resbale. El musgo es un elemento muy común en los árboles de la Gran Bretaña, debido al alto grado de humedad de sus bosques. Este es, pues, un elemento que no se recupera en la traducción. A pesar de la naturalidad del texto encontramos un texto un poco forzado en el cuarto verso de la primera estrofa: la traductora siempre habla de en Jaume, menos aquí que solo dice el nombre sin el artículo delante.

En la segunda estrofa la traductora mantiene la personificación del árbol: tanto en inglés como en catalán el árbol ríe. El original inglés nos habla de la tierra donde ha crecido este árbol; en cambio, la traducción 
al catalán nos habla más del árbol, de cómo son las ramas. En este caso pequeñas, puesto que habla de ramas pequeñas (branquillons), y del estado del árbol: lleno de frutos.

La tercera estrofa introduce a la madre del protagonista, y esto lo encontramos tanto en inglés como en catalán. Es un hecho bastante natural que en una narración donde sale un niño pequeño, inocente, pero a la vez travieso, que trepa por los árboles, aparezca el personaje protector de la madre. La madre trae las manzanas al mercado del pueblo, y se sobreentiende que es para venderlas.

Finalmente, la persona que nos explica la historia nos da la moraleja. En la versión inglesa (ditty), el narrador de la historia nos da un consejo. En catalán el narrador habla de una lección. Esto quiere decir de maestro a discípulo, de padres a hijos, de un superior a un subordinado. La lección aquelll qui bada cau es un refrán catalán popular de Menorca $^{29}$ y popular en Vic. El original inglés también utiliza un refrán que encontramos en nuestro refranero popular: Qui més amunt puja, de més amunt $c a u,{ }^{30}$ pero en este caso la traductora ha optado por utilizar un refrán diferente, a pesar de que mantiene el cariz popular que le da la utilización de un refrán.

La cuarta estrofa acaba de manera diferente. Mientras en inglés el hecho de caer de muy alto sería una lástima, la versión catalana dice que el que cae porque está embobado da risa.

\section{Cantabilidad}

En la parte superior de la partitura en inglés el autor de la música nos explica cómo quiere que se interprete y escribe que quiere que sea alegre (gaily). El autor musical insinúa una notación que hace fácil la utilización de más o menos sílabas en los versos, puesto que en el mismo original así pasa, según conviene en el texto de cada estrofa. Este hecho hace más fácil la adaptación del texto en catalán, para poder conseguir una cantabilidad adecuada manteniendo los acentos prosódicos y musicales correctamente colocados. Este recurso es utilizado ya en el inicio,

\footnotetext{
${ }^{29}$ Alcover y Moll, Diccionari Català-Valencià-Balear, vol 2, 201.

${ }^{30}$ Alcover y Moll, Diccionari Català-Valencià-Balear, vol 3, 66.
} 
en el primer verso de la primera estrofa, por parte de Enriqueta Anglada, que introduce una sílaba de más, pero mantiene el acento principal en la segunda palabra, Jaume. En inglés no se necesita el artículo ante un nombre propio, en cambio sí que se necesita en catalán. La autora del texto catalán puede empezar con la introducción del personaje sin malograr la canción, puesto que el mismo músico ya ha previsto la utilización del inicio anacrúsico del compás anterior para la introducción del primer verso de la cuarta estrofa. Lo mismo se reproduce en el quinto verso de la primera estrofa.

La autora del texto catalán resalta algunas palabras en el mismo lugar donde así también aparecían en el texto original. Ejemplos: pomer, pomes, riure, mare, mercat.

Tommy climbed the apple tree

For to pluck some apples

The tree it shook with laughter

Tommy's mother gathered up

Took them tono the market town
En Jaume puja al pomer

per abastar pomes

l'arbre es posa a riure

Però la mare va a collir

i les portarà al mercat

\section{CONCLUSIÓN}

La traductología nos aporta diversos parámetros necesarios para poder obtener una traducción cantable y adaptada totalmente a la lengua de recepción, con claras prioridades musicales, de manera que al cantar la canción ni siquiera te des cuenta de que la canción pertenece originalmente a otra lengua. Los traductores proporcionan un tipo de traducción mediante la cual el texto traducido «suena» igual que el original. Al mismo tiempo, procuran expresar la misma idea a pesar de utilizar palabras o expresiones diferentes. Finalmente también han tomado en cuenta no alterar la métrica, ${ }^{31}$ aunque se alejan totalmente de la traducción literal. Estas traducciones que hemos mostrado utilizan diferentes maneras de traducir, pero siempre con claras prioridades musicales.

\footnotetext{
${ }^{31}$ Ignacio Pérez, «Estudio del fenómeno traductor en la canción moderna: Bon Jovi en castellano», en Últimas Corrientes Teóricas en los Estudios de Traducción y sus Aplicaciones eds. Ann Barr, María Rosario Martin Ruano y Jesús Torres del Rey (Salamanca: Universidad de Salamanca: 2001).
} 
La traducción de canciones infantiles nos permite por un lado conocer la cultura, el vocabulario y las tradiciones del texto de salida; y, por otro lado, el transporte de este vocabulario, frases hechas y expresiones populares de la lengua de llegada. La traducción hace que nos demos cuenta de que el mundo es muy diverso, pero que también hay muchos elementos que podemos compartir. Las corales infantiles catalanas interpretan canciones en su propia lengua, así como canciones traducidas de otras lenguas al catalán.

Las corales infantiles a través del SCIC llenaron el espacio vacío de la enseñanza de la música en los centros escolares de la época de la represión franquista, procurando el uso del catalán por parte de los niños y niñas a través de la canción. Las corales infantiles, con la ayuda de sus directores, buscaron la manera de transmitir los valores del gusto por la música, ya que la música como asignatura no se contemplaba en la enseñanza primaria después de la Guerra Civil española. Los directores que iniciaron el movimiento SCIC fueron herederos de la Escola Moderna, la cual utilizaba métodos educativos que proponían al alumno como agente activo de la propia educación.

A partir de la traducción podemos conocer las particularidades de otras lenguas, conocer el folclore de otros países. En definitiva, abrir una ventana al mundo que permita a los niños conocer otras realidades mediante su propia lengua. Con la traducción de canciones podemos introducir peculiaridades de otras culturas a la nuestra, y de este modo favorecer y ampliar los conocimientos generales de los niños y las niñas a través de la música.

\section{Nota sobre la autora:}

Núria Medina Casanovas es especialista en la investigación sobre el trabajo interdisciplinario de música e inglés y los efectos motivadores del trabajo conjunto de estas asignaturas. Desde 1988 es profesora de Didáctica de la Lengua Inglesa, Lengua para Usos Académicos y Enfoques y Metodología para la Enseñanza del Inglés en la Facultat d’Educació Traducció i Ciències Humanes de la Universitat de Vic-Universitat Central de Catalunya. Actualmente forma parte del grupo de investigación GRELL. 
Es Licenciada en Filología Inglesa por la Universitat de Barcelona y Doctora en Traducción, Lenguas y Literaturas por la UVIC-UCC con el trabajo English Rap: Una proposta interdisciplinària d'anglès i de música per a Primària. Ha publicado diversos libros de texto para la enseñanza del inglés en educación primaria, diferentes artículos sobre la importancia de la música para la enseñanza del inglés como lengua extranjera y el Easy English Dictionary (Eumo Editorial, 2004).

Ha llevado a cabo diferentes seminarios sobre los aspectos de la didáctica del inglés y participado en diversos congresos sobre la enseñanza de este idioma como lengua extranjera. Ha sido miembro del comité organizador del congreso HEPCLIL y organizado diversas jornadas sobre la enseñanza del inglés en infantil y primaria en la UVIC-UCC. 


\section{ANEXO 1}

\section{CUCKOO!}

Benjamin Britten

Del ciclo Friday Afternoons

Texto de Jane Taylor

De Tom Tiddler's Ground

Antologia de poemas a cargo de

Walter de la Mare

Cuckoo, Cuckoo, What do you do?

«In April I open my bill;

In May I sing night and day;

In June I change my tune;

In July far-far I fly;

In August away I must.»

Cuckoo, cuckoo, cuckoo!

\section{CU-CUT! \\ Josep Vigo 1994}

Cucut, cucut! Plou i és eixut.

«L'abril, ves!, fa poc que n'he après.

El maig ja canto a bell raig.

El juny tothom qui em sent

diu que entono malament.

Sabeu, doncs, què faig? Me'n vaig.»

Cucut, cucut, cucut

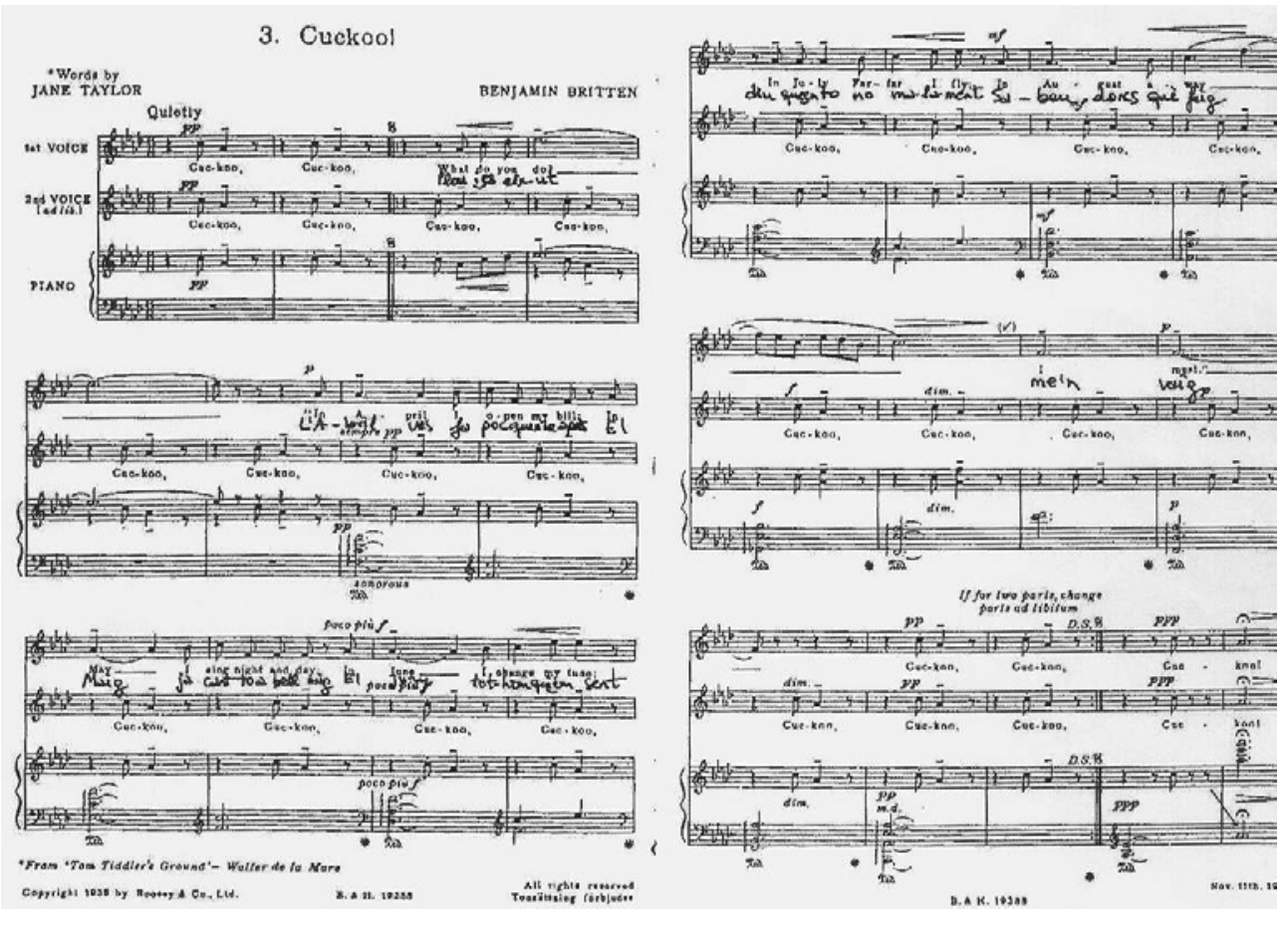




\section{ANEXO 2}

\section{TOMMY AND THE APPLES}

\section{Canción de Gales.}

Armonización: S. Northcote

Tommy climbed the apple tree

For to pluck some apples;

Tree with moss was overgrown,

Tom fell down,

Down, down,

Tommy and the apples.

And as Tom was falling down

The tree it shook with laughter;

Shook the apples to the ground

(Lumpy ground)

Down, down,

And Tom came tumbling after.

Tommy's mother gathered up

The apples in a basket;

Took them to the market town

(Busy town),

Down, down,

Took them to the market.

Now boys and girls take my advice,

For ending of my ditty:

Don't go climbing up too high,

Or you'll fall,

Down, down,

And that would be a pity.
EL POMER.

Adaptación: E. Anglada1991

En Jaume puja al pomer per abastar pomes.

Com que és un xic babau

Jaume cau,

cau, cau

en Jaume i les pomes.

Mentre en Jaume va caient

l'arbre es posa a riure espolsant els branquillons

plens de fruits,

fruits, fruits,

sobre el pobre Jaume.

Però la mare va a collir

totes les pomes

i les portarà al mercat

al mercat,

cat, cat,

al mercat de la vila.

Aprengueu bé la lliçó

aprengueu a viure,

perquè aquell qui bada cau

bada cau,

cau, cau,

i a tothom fa riure. 


\section{TOMMY AND THE APPLES}

H. H.

Welsh Nursery Song * Arranged by S.N.

voICE

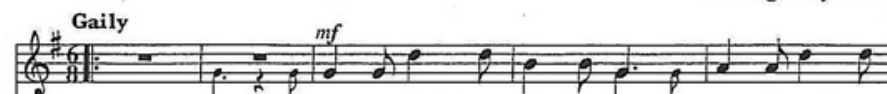

1. Tom-my climbed the ap - ple tree For to plucksome

2. And as Tom was fall-ingdown The tree it shookwith
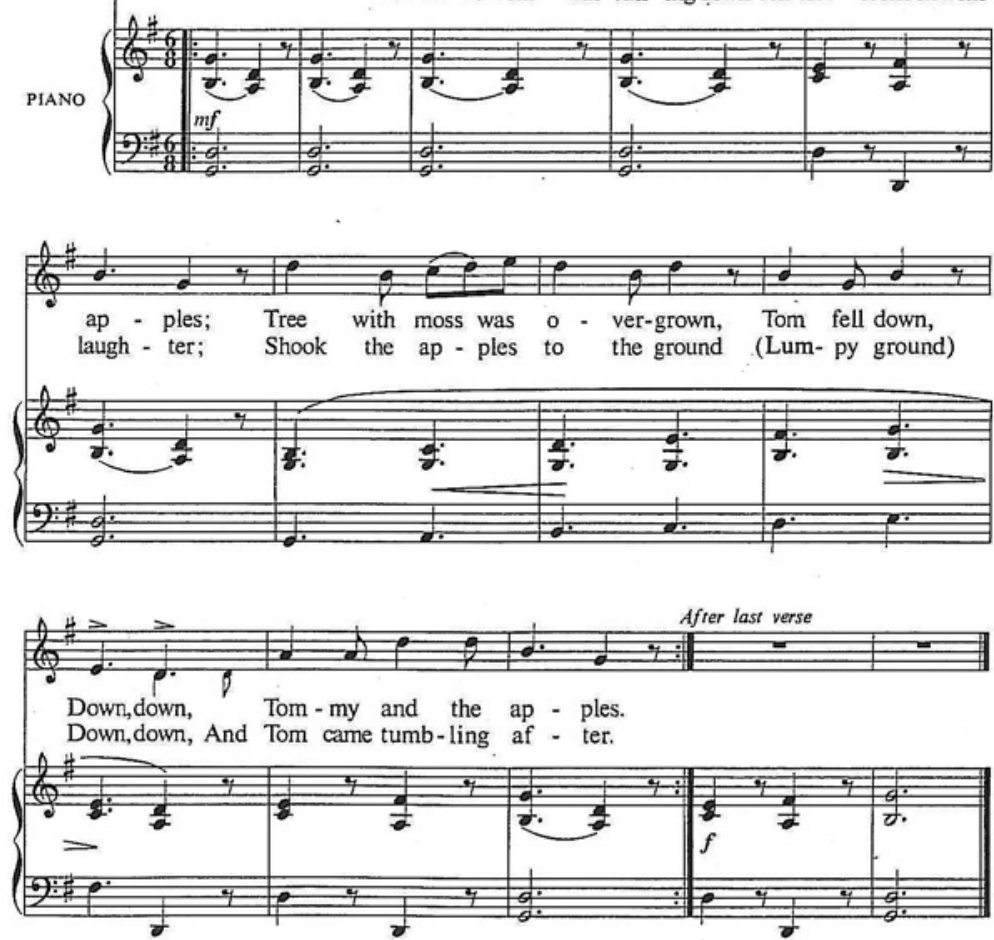

3. Tommy's mother gathered up

The apples in a basket;

Took them to the market town

(Busy town),

Down, down,

Took them to the market.

4. Now boys and girls take my advice,

For ending of my ditty:

Don't go climbing up too high,

Or you'll fall,

Down, down,

And that would be a pity.

* Noted by the arranger from the singing of his uncle, the late Darran Llewellyn. 


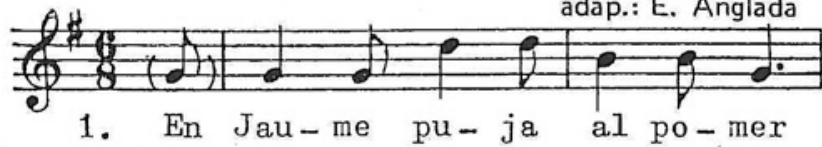

1. En Jau-me pu-ja a.l po-mer

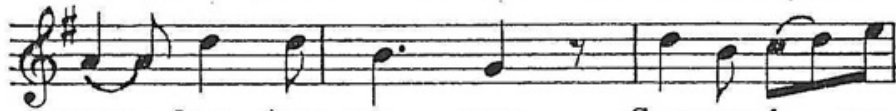

per a-bas-tar po - mes. Comque és

un
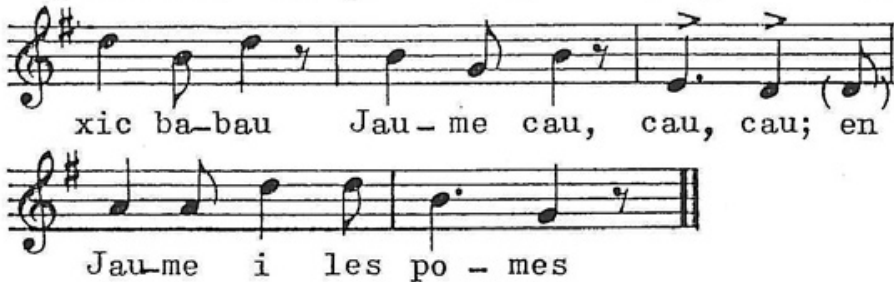

2. Mentre en Jaume va caient

l'arbre es posa a riure espolsant els branquillons plens de fruits, fruits, fruits,

3. Però la mare va acollir totes les pomes

i les portarà al mercat al mercat, cat, cat, al mercat de la vila.

4. Aprengueu bé la lliçó aprengueu a viure, perquè aquell qui bada cau bada cau, cau, cau, i a tothom fa riure. 